\title{
Detecting Sepsis: Are Two Opinions Better Than One?
}

\author{
Poushali Bhattacharjee, MD¹, Matthew M. Churpek, MD, MPH, PhD², Ashley Snyder, MPH¹, Michael D. Howell, MD, MPH², \\ Dana P. Edelson, MD, MS ${ }^{1 *}$
}

'Section of Hospital Medicine and 'Section of Pulmonary and Critical Care Medicine, Department of Medicine, University of Chicago Medical Center, Chicago, Illinois.

The diagnosis of sepsis requires that objective criteria be met with a corresponding subjective suspicion of infection. We conducted a study to characterize the agreement between different providers' suspicion of infection and the correlation with patient outcomes using prospective data from a general medicine ward. Registered nurse (RN) suspicion of infection was collected every 12 hours and compared with medical doctor or advanced practice professional (MD/APP) suspicion, defined as an existing order for antibiotics or a new order for blood or urine cultures within the 12 hours before nursing screen time. During the study period, 1386 patients yielded 11,489 screens, $3744(32.6 \%)$ of which met at least 2 systemic inflammatory response syndrome (SIRS) criteria. Infection was suspected by RN and MD/APP in $5.8 \%$ of cases, by RN only in $22.2 \%$, by MD/APP only in $7.2 \%$, and by neither provider in $64.7 \%$. Overall agreement rate was $80.7 \%$ for suspicion of infection ( $\kappa=0.11, P<0.001$ ). Progression to severe sepsis or shock was highest when both providers suspected infection in a SIRS-positive patient (17.7\%), was substantially reduced with single-provider suspicion (6.0\%), and was lowest when neither provider suspected infection $(1.5 \%)(P<$ $0.001)$. Provider disagreement regarding suspected infection is common, with RNs suspecting infection more often, suggesting that a collaborative model for sepsis detection may improve timing and accuracy. Journal of Hospital Medicine 2017;12:256-258. (C) 2017 Society of Hospital Medicine
Sepsis is a leading cause of hospital mortality in the United States, contributing to up to half of all deaths. ${ }^{1}$ If the infection is identified and treated early, however, its associated morbidity and mortality can be significantly reduced. ${ }^{2}$ The 2001 sepsis guidelines define sepsis as the suspicion of infection plus meeting 2 or more systemic inflammatory response syndrome (SIRS) criteria. $^{3}$ Although the utility of SIRS criteria has been extensively debated, providers' accuracy and agreement regarding suspicion of infection are not yet fully characterized. This is very important, as the source of infection is often not identified in patients with severe sepsis or septic shock. ${ }^{4}$

Although much attention recently has been given to ideal objective criteria for accurately identifying sepsis, less is known about what constitutes ideal subjective criteria and who can best make that assessment. ${ }^{5-7}$ We conducted a study to measure providers' agreement regarding this subjective assessment and the impact of that agreement on patient outcomes.

\section{METHODS}

We performed a secondary analysis of prospectively collected data on consecutive adults hospitalized on a general med-

\footnotetext{
*Address for correspondence and reprint requests: Dana P. Edelson, MD, MS, Section of Hospital Medicine, Department of Medicine, University of Chicago Medical Center, 5841 S Maryland Ave, W312, Chicago, IL 60637; Telephone: 773-834-2191; Fax: 773-795-7398; E-mail: dperes@medicine.bsd.uchicago.edu Additional Supporting Information may be found in the online version of this article.

Received: January 22, 2016; Revised: August 22, 2016; Accepted: August 31, 2016
}

2017 Society of Hospital Medicine DOI 10.12788/jhm.2721 icine ward at an academic medical center between April 1, 2014 and March 31, 2015. This study was approved by the University of Chicago Institutional Review Board with a waiver of consent.

A sepsis screening tool was developed locally as part of the Surviving Sepsis Campaign Quality Improvement Learning Collaborative $^{8}$ (Supplemental Figure). This tool was completed by bedside nurses for each patient during each shift. Bedside registered nurse $(\mathrm{RN})$ suspicion of infection was deemed positive if the nurse answered yes to question 2: "Does the patient have evidence of an active infection?" We compared RN assessment with assessment by the ordering provider, a medical doctor or advanced practice professionals (MD/APP), using an existing order for antibiotics or a new order for either blood or urine cultures placed within 12 hours before nursing screen time to indicate MD/APP suspicion of infection.

All nursing screens were transcribed into an electronic database, excluding screens not performed, or missing $\mathrm{RN}$ suspicion of infection. For quality purposes, screening data were merged with electronic health record data to verify SIRS criteria at the time of the screens as well as the presence of culture and/or antibiotic orders preceding the screens. Outcome data were obtained from an administrative database and confirmed by chart review using the 2001 sepsis definitions. ${ }^{6}$ Data were de-identified and time-shifted before this analysis. SIRS-positive criteria were defined as meeting 2 or more of the following: temperature higher than $38^{\circ} \mathrm{C}$ or lower than $36^{\circ} \mathrm{C}$; heart rate higher than 90 beats per minute; respiratory rate more than 20 breaths per minute; and white blood cell count more than $2,000 / \mathrm{mm}^{3}$ or less than $4,000 / \mathrm{mm}^{3}$. 
The primary clinical outcome was progression to severe sepsis or septic shock. Secondary outcomes included transfer to intensive care unit (ICU) and in-hospital mortality. Given that RN and MD/APP suspicion of infection can vary over time, only the initial screen for each patient was used in assessing progression to severe sepsis or septic shock and in-hospital mortality. All available screens were used to investigate the association between each provider's suspicion of infection over time and ICU transfer.

Demographic characteristics were compared using the $\chi^{2}$ test and analysis of variance, as appropriate. Provider agreement was evaluated with a weighted $\kappa$ statistic. Fisher exact tests were used to compare proportions of mortality and severe sepsis/septic shock, and the McNemar test was used to compare proportions of ICU transfers. The association of outcomes based on provider agreement was evaluated with a nonparametric test for trend.

\section{RESULTS}

During the study period, 1386 distinct patients had 13,223 screening opportunities, with a $95.4 \%$ compliance rate. A total of 1127 screens were excluded for missing nursing documentation of suspicion of infection, leaving 1192 first screens and 11,489 total screens for analysis. Of the completed screens, 3744 (32.6\%) met SIRS criteria; suspicion of infection was noted by both RN and MD/APP in $5.8 \%$ of cases, by RN only in $22.2 \%$, by MD/APP only in $7.2 \%$, and by neither provider in $64.7 \%$ (Figure 1). Overall agreement rate was $80.7 \%$ for suspicion of infection $(\kappa=0.11, P<0.001)$. Demographics by subgroup are shown in the Supplemental Table. Progression to severe sepsis or shock was highest when both providers suspected infection in a SIRS-positive patient $(17.7 \%)$, was substantially reduced with single-provider suspicion $(6.0 \%)$, and was lowest when neither provider suspected infection $(1.5 \%)(P<0.001)$. A similar trend was found for in-hospital mortality (both providers, $6.3 \%$; single provider, $2.7 \%$; neither provider, $2.5 \% ; P=0.01$ ). Compared with $\mathrm{MD} /$ APP-only suspicion, SIRS-positive patients in whom only RNs suspected infection had similar frequency of progression to severe sepsis or septic shock ( $6.5 \%$ vs $5.6 \% ; P=0.52)$ and higher mortality $(5.0 \%$ vs $1.1 \% ; P=0.32)$, though these findings were not statistically significant.

For the 121 patients (10.2\%) transferred to ICU, RNs were more likely than MD/APPs to suspect infection at all time points (Figure 2). The difference was small $(P=0.29) 48$ hours before transfer (RN, 12.5\%; MD/APP, $5.6 \%$ ) but became more pronounced $(P=0.06)$ by 3 hours before transfer (RN, 46.3\%; MD/APP, 33.1\%). Nursing assessments were not available after transfer, but 3 hours after transfer the proportion of patients who met MD/APP suspicion-of-infection criteria $(44.6 \%)$ was similar $(P=0.90)$ to that of the $R N_{s} 3$ hours before transfer $(46.3 \%)$.

\section{DISCUSSION}

Our findings reveal that bedside nurses and ordering providers routinely have discordant assessments regarding presence

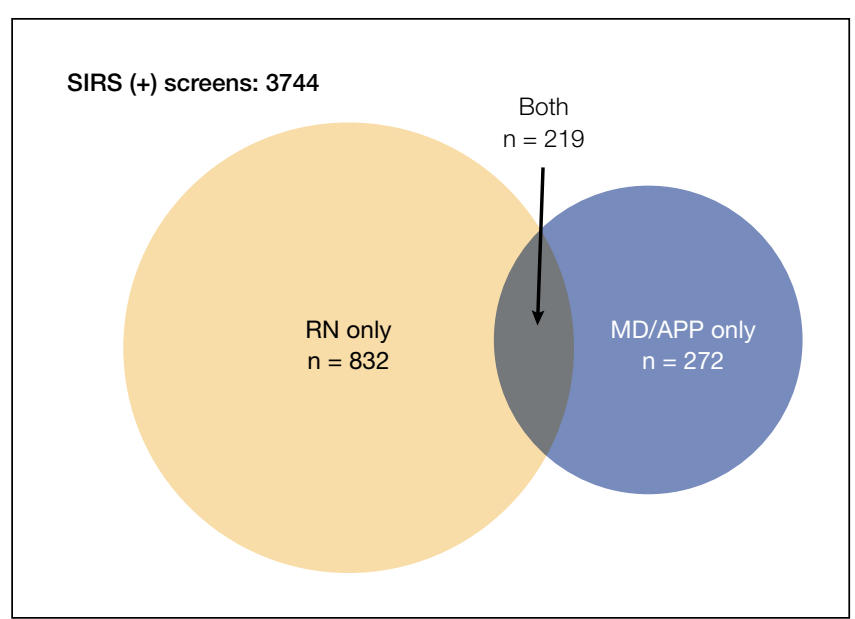

FIG. 1. Provider agreement on suspicion of infection in patients meeting 2 of 4 SIRS criteria.

NOTE: Abbreviations: APP, advanced practice professional; MD, medical doctor; RN, registered nurse; SIRS, systemic inflammatory response syndrome.

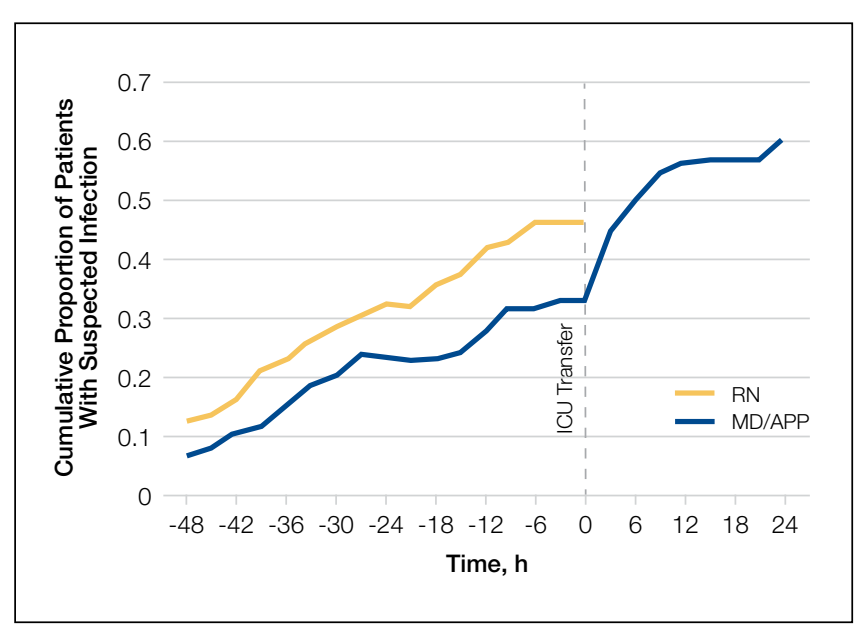

FIG. 2. Cumulative suspicion of infection by provider over time in patients transferred to ICU.

NOTE: Abbreviations: APP, advanced practice professional; ICU, intensive care unit; MD, medical doctor; RN, registered nurse.

of infection. Specifically, when RNs are asked to screen patients on the wards, they are suspicious of infection more often than MD/APPs are, and they suspect infection earlier in ICU transfer patients. These findings have significant implications for patient care, compliance with the new national SEP-1 Centers for Medicare \& Medicaid Services quality measure, and identification of appropriate patients for enrollment in sepsis-related clinical trials.

To our knowledge, this is the first study to explore agreement between bedside RN and MD/APP suspicion of infection in sepsis screening and its association with patient outcomes. Studies on nurse and physician concordance in other domains have had mixed findings. ${ }^{9-11}$ The high discordance rate found in our study points to the highly subjective nature of suspicion of infection.

Our finding that RNs suspect infection earlier in patients 
transferred to ICU suggests nursing suspicion has value above and beyond current practice. A possible explanation for the higher rate of RN suspicion, and earlier RN suspicion, is that bedside nurses spend substantially more time with their patients and are more attuned to subtle changes that often occur before any objective signs of deterioration. This phenomenon is well documented and accounts for why rapid response calling criteria often include "nurse worry or concern." 12,13 Thus, nurse intuition may be an important signal for early identification of patients at high risk for sepsis.

That about one third of all screens met SIRS criteria and that almost two thirds of those screens were not thought by $\mathrm{RN}$ or MD/APP to be caused by infection add to the literature demonstrating the limited value of SIRS as a screening tool for sepsis. ${ }^{14}$ To address this issue, the 2016 sepsis definitions propose using the quick Sepsis-Related Organ Failure Assessment (qSOFA) to identify patients at high risk for clinical deterioration; however, the Surviving Sepsis Campaign continues to encourage sepsis screening using the SIRS criteria. ${ }^{15}$

Limitations of this study include its lack of generalizability, as it was conducted with general medical patients at a single center. Second, we did not specifically ask the MD/ APPs whether they suspected infection; instead, we relied on their ordering practices. Third, RN and MD/APP assessments were not independent, as RNs had access to MD/APP orders before making their own assessments, which could bias our results.

Discordance in provider suspicion of infection is common, with RNs documenting suspicion more often than MD/APPs, and earlier in patients transferred to ICU. Suspicion by either provider alone is associated with higher risk for sepsis progression and in-hospital mortality than is the case when neither provider suspects infection. Thus, a collaborative method that includes both RNs and MD/APPs may improve the accuracy and timing of sepsis detection on the wards.

\section{Acknowledgments}

The authors thank the members of the Surviving Sepsis Campaign (SSC) Quality Improvement Learning Collaborative at the University of Chicago for their help in data collection and review, especially Meredith Borak, Rita Lanier, Mary Ann Francisco, and Bill Marsack. The authors also thank Thomas Best and Mary-Kate Springman for their assistance in data entry and Nicole Twu for administrative support. Data from this study were provided by the Clinical Research Data Warehouse
(CRDW) maintained by the Center for Research Informatics (CRI) at the University of Chicago. CRI is funded by the Biological Sciences Division of the Institute for Translational Medicine/Clinical and Translational Science Award (CTSA) (National Institutes of Health UL1 TR000430) at the University of Chicago.

Disclosures: Dr. Bhattacharjee is supported by postdoctoral training grant 4T32HS000078 from the Agency for Healthcare Research and Quality. Drs. Churpek and Edelson have a patent pending (ARCD.P0535US.P2) for risk stratification algorithms for hospitalized patients. Dr. Churpek is supported by career development award K08 HL121080 from the National Heart, Lung, and Blood Institute. Dr. Edelson has received research support from Philips Healthcare (Andover, Massachusetts), American Heart Association (Dallas, Texas), and Laerdal Medical (Stavanger, Norway) and has ownership interest in Quant HC (Chicago, Illinois), which is developing products for risk stratification of hospitalized patients. The other authors report no conflicts of interest.

\section{References}

1. Liu V, Escobar GJ, Greene JD, et al. Hospital deaths in patients with sepsis from 2 independent cohorts. JAMA. 2014;312(1):90-92.

2. Rivers E, Nguyen B, Havstad S, et al; Early Goal-Directed Therapy Collaborative Group. Early goal-directed therapy in the treatment of severe sepsis and septic shock. N Engl J Med. 2001;345(19):1368-1377.

3. Levy MM, Fink MP, Marshall JC, et al; SCCM/ESICM/ACCP/ATS/SIS. 2001 SCCM/ESICM/ACCP/ATS/SIS International Sepsis Definitions Conference. Crit Care Med. 2003;31(4):1250-1256.

4. Vincent JL, Sakr Y, Sprung CL, et al; Sepsis Occurrence in Acutely Ill Patients Investigators. Sepsis in European intensive care units: results of the SOAP study. Crit Care Med. 2006;34(2):344-353.

5. Kaukonen KM, Bailey M, Pilcher D, Cooper DJ, Bellomo R. Systemic inflammatory response syndrome criteria in defining severe sepsis. $N$ Engl J Med. 2015;372(17):1629-1638

6. Vincent JL, Opal SM, Marshall JC, Tracey KJ. Sepsis definitions: time for change. Lancet. 2013;381(9868):774-775.

7. Singer M, Deutschman CS, Seymour CW, et al. The Third International Consensus Definitions for Sepsis and Septic Shock (Sepsis-3). JAMA. 2016;315(8):801-810.

8. Surviving Sepsis Campaign (SSC) Sepsis on the Floors Quality Improvement Learning Collaborative. Frequently asked questions (FAQs). Society of Critical Care Medicine website. http://www.survivingsepsis.org/SiteCollectionDocuments/About-Collaboratives.pdf. Published October 8, 2013

9. Fiesseler F, Szucs P, Kec R, Richman PB. Can nurses appropriately interpret the Ottawa ankle rule? Am J Emerg Med. 2004;22(3):145-148.

10. Blomberg H, Lundström E, Toss H, Gedeborg R, Johansson J. Agreement between ambulance nurses and physicians in assessing stroke patients. Acta Neurol Scand. 2014;129(1):4955.

11. Neville TH, Wiley JF, Yamamoto MC, et al. Concordance of nurses and physicians on whether critical care patients are receiving futile treatment. Am J Crit Care. 2015;24(5):403410.

12. Odell M, Victor C, Oliver D. Nurses' role in detecting deterioration in ward patients: systematic literature review. J Adv Nurs. 2009;65(10):1992-2006.

13. Howell MD, Ngo L, Folcarelli P, et al. Sustained effectiveness of a primary-team-based rapid response system. Crit Care Med. 2012;40(9):2562-2568.

14. Churpek MM, Zadravecz FJ, Winslow C, Howell MD, Edelson DP. Incidence and prognostic value of the systemic inflammatory response syndrome and organ dysfunctions in ward patients. Am J Respir Crit Care Med. 2015;192(8):958-964.

15. Antonelli M, DeBacker D, Dorman T, Kleinpell R, Levy M, Rhodes A; Surviving Sepsis Campaign Executive Committee. Surviving Sepsis Campaign responds to Sepsis-3. Society of Critical Care Medicine website. http://www.survivingsepsis. org/SiteCollectionDocuments/SSC-Statements-Sepsis-Definitions-3-2016.pdf. Published March 1, 2016. Accessed May 11, 2016. 